\title{
MEDIAS MÓVILES, COMO ALTERNATIVAS DE INVERSIÓN EN UN ÍNDICE ACCIONARIO.
}

\author{
MOVING AVERAGES, AS INVESTMENT ALTERNATIVES IN A \\ STOCK INDEX.
}

\author{
Mtro. Gerardo Ablanedo Rosas a \\ Mtra. Lágrima de María Azcárraga Montiel ${ }^{b}$ \\ Dr. Edmundo Mejía Sánchez ${ }^{\mathrm{c}}$
}

\author{
a Benemérita Universidad Autónoma de Puebla \\ Facultad de Contaduría Pública, gablanedo@gmail.com \\ ${ }^{\mathrm{b}}$ Universidad del Valle de México \\ Facultad de Negocios, nutriologazcarraga@gmail.com \\ ${ }^{c}$ Benemérita Universidad Autónoma de Puebla \\ Facultad de Contaduría Pública, amenodan@hotmail.com
}

\section{RESUMEN}

En inversión accionaria, identificar la tendencia del precio es importante para tomar decisiones de compra o venta de títulos o valores bursátiles. Dentro del análisis técnico, existen métodos que intentan predecir el comportamiento de un valor en función a datos históricos. En este trabajo se analizan dos estrategias de análisis técnico: la media móvil exponencial y la media móvil de convergencia y divergencia. Se generan datos históricos y se observa su comportamiento en el tiempo. Las estrategias se aplican a precios históricos del índice accionario del país Singapur que, por su movimiento lateral, es difícil de predecir y las medias móviles serían la herramienta que apoyaría la decisión del inversor. Además, y para fundamentar las conclusiones de éste trabajo, los resultados que se obtienen se comparan con la estrategia "buy and hold" que corresponde a comprar y mantener durante largo plazo y se exponen los efectos. Este trabajo es una investigación cuantitativa basada en el método empírico-analítico, que muestra las consecuencias de aplicar la media móvil exponencial y la media móvil de convergencia y divergencia a gráficos diarios del índice accionario mencionado. Se pretende identificar la herramienta de mayor funcionalidad que reconozca el mayor número de aciertos con posiciones largas y cortas, lo que permitiría incrementar los rendimientos de inversiones especulativas y con ello orientar al inversionista sobre la mejor estrategia. Los resultados basados en pruebas de back testing demuestran que, en ocasiones, el análisis técnico y el uso de medias móviles que son herramientas sofisticadas de trading, no generan altos rendimientos y que la estrategia más sencilla basada en comprar y esperar puede ser más rentable en el largo plazo. 
PALABRAS CLAVE: Análisis técnico; Media móvil exponencial; Media móvil de convergencia y divergencia (MACD); Tendencia.

\section{ABSTRACT}

In stock investment, identifying the price trend is important to make decisions to buy or sell securities or securities. Within the technical analysis, there are methods that try to predict the behavior of a value based on historical data. In this paper, two technical analysis strategies are analyzed: the exponential moving average and the moving convergence and divergence average. Historical data are generated and its behavior over time is observed. The strategies are applied to historical prices of the stock index of the country Singapore, which, due to its lateral movement, is difficult to predict and the moving averages would be the tool that would support the decision of the investor. In addition, and to base the conclusions of this work, the results obtained are compared with the "buy and hold" strategy that corresponds to buying and maintaining for a long term and the effects are exposed. This work is a quantitative research based on the empirical-analytical method, which shows the consequences of applying the exponential moving average and the moving average of convergence and divergence to daily charts of the aforementioned stock index. The aim is to identify the tool with the most functionality that recognizes the greatest number of successes with long and short positions, which would allow to increase the yields of speculative investments and thereby guide the investor on the best strategy. The results based on back testing prove that, at times, technical analysis and the use of mobile averages that are sophisticated trading tools do not generate high returns and that the simpler strategy based on buying and waiting can be more profitable in the long-term.

Keywords: Technical analysis; Exponential moving average; Moving average of convergence and divergence (MACD); Trend.

\section{I.- INTRODUCCIÓN}

Encontrar la estrategia adecuada incorporando análisis técnico en los precios de activos financieros al momento de invertir en los índices accionarios, ha sido una labor de investigación por mucho tiempo. El objetivo es tratar de maximizar las ganancias en los mercados financieros que se encuentran en constante movimiento o tendencia. Muchos de los trabajos relacionados con éste tema se enfocan a la inclusión de medias móviles para tratar de identificar la dirección del precio. No obstante, no se realizan pruebas de back testing que permitan identificar si dichas estrategias son rentables en el largo plazo. En este trabajo se hace una propuesta cuantitativa y comparativa sobre los beneficios esperados de invertir adoptando las medias móviles.

La estrategia "Buy and hold" se refiere a comprar un activo financiero y esperar a que 
en periodos prolongados de tiempo se obtengan resultados favorables. Es una estrategia muy respetada por muchos inversionistas. Su fundamento se basa en que la renta variable es un tipo de inversión que en el largo plazo se comporta muy bien porque recompensa con buenos rendimientos y más cuando existe una buena diversificación (Sánchez, G.J. 2016). También se justifica porque el hecho de realizar varias operaciones de entrada y salida del mercado implica gasto en comisiones que disminuyen el beneficio. Finalmente se argumenta que en el largo plazo los precios de los activos financieros siempre suben, y comprar y mantener la posición implica que en periodos prolongados de tiempo se generen beneficios (Maidona, A.D. 2016). Sin embargo, en mercados laterales, la estrategia "Buy and hold" no es la adecuada, debido a que el precio se mantiene en un rango durante largo plazo lo que implica escaso o nulo crecimiento de la inversión (Murphy, J.J. 2005).

Las medias móviles son herramientas de análisis técnico que pretenden anticipar la dirección del mercado que puede ser alcista, bajista o lateral. Algunos inversores pretenden basarse en estas técnicas, pero desconocen las consecuencias en términos de rendimiento. Esta investigación, presenta los resultados comparativos por aplicar la media móvil exponencial y media móvil de convergencia a un índice accionario e identifica sus beneficios. Posteriormente, se comparan contra la técnica más antigua del mercado basada en comprar un título y esperar ("buy and hold") y se muestran nuevamente los resultados. La intención es ofrecer una idea clara en términos de rendimiento sobre las ventajas y desventajas de invertir tratando de predecir la tendencia aplicando análisis técnico.

\section{II.- PLANTEAMIENTO DEL PROBLEMA}

El problema general que se presenta para cualquier inversor, es identificar la estrategia de comercialización más adecuada en términos de rentabilidad, aplicada a los precios históricos de un instrumento que se encuentre o no en tendencia. En este trabajo se realiza la investigación sobre datos que corresponden a un índice accionario con movimiento lateral de largo plazo como es el de Singapur, pero cuya lateralidad presenta tendencias alcistas y bajistas de corto plazo, lo que implica un segundo dilema que está relacionado con la incorporación de una media móvil exponencial o una media móvil de convergencia y divergencia, ambas en el marco de tiempo de un día y con los mismos parámetros de valor promedio con el objetivo de tratar de incrementar los beneficios al incorporar al inversor en la tendencia.

Los dilemas a resolver se enfocan a dar respuesta a:

- ¿ ¿Cuál de las estrategias es la más aceptable en términos de rendimiento?

- ¿Cuáles son los inconvenientes de adoptar la estrategia más rentable? 
- El uso de media móvil supera la estrategia "buy and hold"

- El análisis técnico permite mejorar los beneficios de trading

En resumen, la problemática se enfoca a distinguir entre la aplicación de la media móvil exponencial y la media móvil de convergencia y divergencia (MACD), y responder a cuál de ellas genera mayores rendimientos y a comparar contra la técnica "Buy and hold" en un mercado lateral.

\section{III.- OBJETIVOS}

Dentro de los objetivos específicos de ésta investigación están:

- Identificar el beneficio esperado de invertir aplicando la media móvil exponencial a un marco temporal diario.

- Mencionar el beneficio esperado de invertir aplicando la media móvil de convergencia y divergencia a un marco temporal diario.

- Determinar el beneficio esperado de invertir, aplicando la técnica "Buy and hold".

- Analizar y comparar los resultados de aplicar cada técnica.

- Señalar la estrategia cuyo poder predictivo arroje los mejores resultados al momento de realizar trading.

\section{IV.- MARCO TEÓRICO CONCEPTUAL}

En este trabajo se considera que el análisis técnico es una herramienta importante para poder predecir la tendencia en los precios de activos bursátiles. Las técnicas expuestas se pueden aplicar a índices accionarios, materias primas, divisas o acciones. Se basa en el análisis del comportamiento histórico de los precios y no se sustenta en el análisis fundamental, por lo tanto, no trata de estimar el precio justo, caro o barato de una acción, índice, divisa o materia prima. Más bien, se intenta predecir la evolución o tendencia futura del precio de un activo (que puede ser alcista o bajista) y no tanto estimar su valor real o esperado.

El fundamento de la inversión bursátil se basa, en primera instancia, en la adquisición o compra de un activo financiero a un precio, cuyo valor debería incrementarse con el transcurso del tiempo, para venderlo en el futuro y obtener un rendimiento. Durante todo este tiempo, el precio estaría sujeto a distinto tipo de tendencias, es decir, subiría y bajaría constantemente de precio, pero a largo plazo generaría beneficios. La idea del análisis técnico es la de identificar las distintas tendencias que se presentan durante el tiempo con el objetivo de buscar mayores beneficios cuando el activo suba y baje de precio, y así, esta herramienta se convierte en elemento de apoyo para 
desarrollar la predicción en lo que se refiere a activos bursátiles.

En este trabajo se mencionarán tres maneras de invertir y los resultados esperados en cada una de ellas.

1. "Buy and hold". Ésta técnica es la más sencilla y la más usada por muchos inversores en el mundo. Se trata de adquirir un activo financiero $y$ simplemente esperar a que se incremente su valor para posteriormente vender. Pero no todos los activos financieros suben de precio, es decir, existen algunos activos cuyo precio se encuentran en rango o con movimiento lateral, esto implica que el precio se mueve en una "banda" y no incrementa su valor sustancialmente. Éste es el caso del mercado accionario de Singapur (ver figura 1), cuyos precios se representan con pequeñas barras verticales que se mueven dentro de un rango lateral dentro de las líneas horizontales de color rojo.

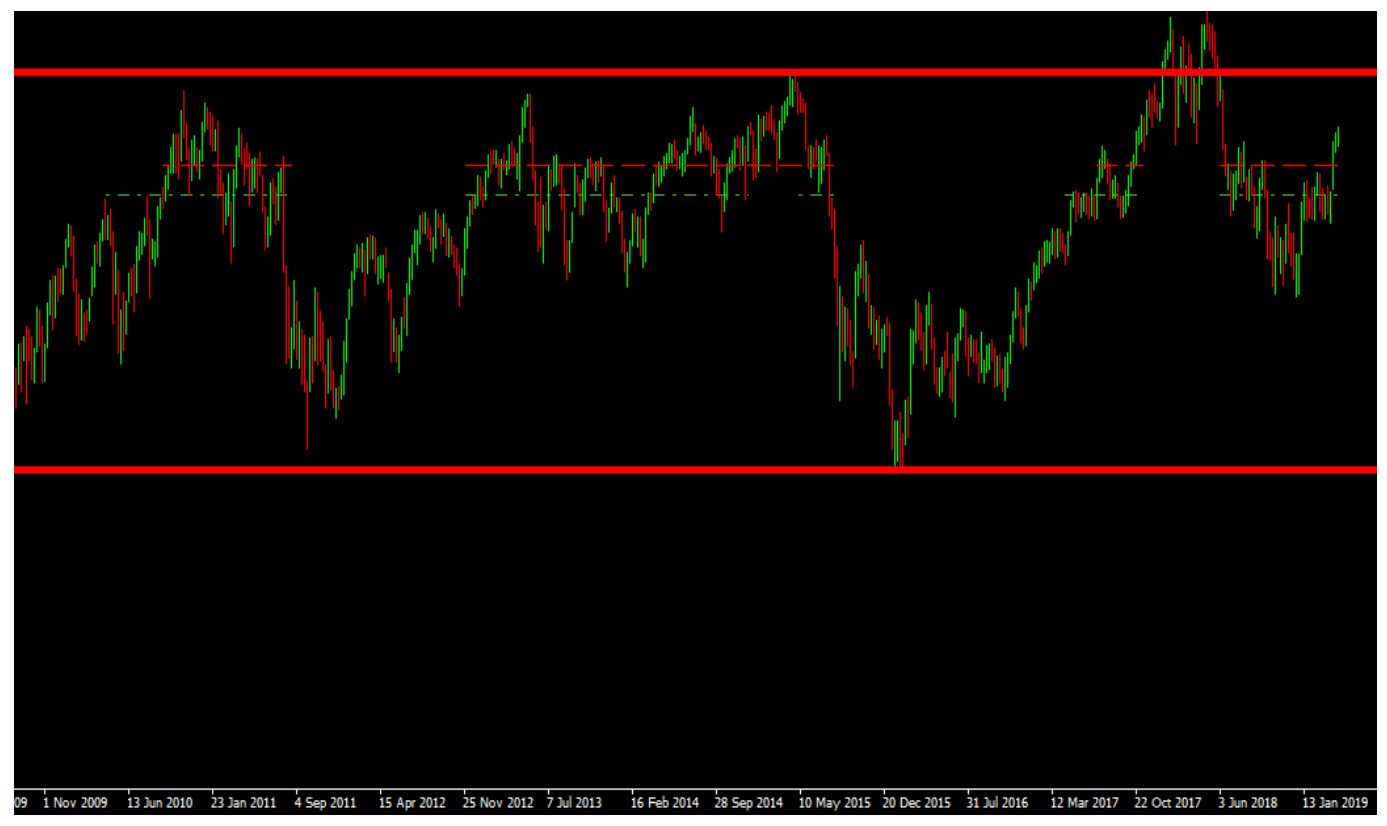

Figura 1. Movimiento accionario del mercado de Singapur. Fuente: Elaboración propia incorporando datos de la plataforma Metatrader 4. (2018)

2. Las medias móviles son indicadores de apoyo para el análisis técnico, la mayoría de los inversionistas las incorporan en sus estrategias debido a su versatilidad, sencillez y practicidad (Rodríguez \& García Fronti, 2013). Resultan de obtener el promedio de un cierto bloque de datos o valores. Por ejemplo, si se busca obtener la media móvil de los precios al cierre de 20 días, se suman los precios de los últimos 20 días y se divide el total entre 20 y se traslada hacia adelante con cada día de operaciones. La forma de 
calcular la media móvil es a partir del total de los precios de cierre de los últimos 20 días. El nuevo cierre de cada día se añade al total y se le resta el cierre de 21 días atrás (Blázquez, 2014).

Para dejar claro cómo se calcula la media móvil simple, en la figura 2 se presenta una serie de precios que corresponden a cifras aleatorias y se calcula la media móvil de 3 periodos (que en el ejemplo conciernen a días). El primer valor que servirá para construir la media móvil es de 101.5 y se obtiene de promediar las cifras: $101 ; 101.5$ y 102 . El segundo valor que corresponde a 102.1 se obtiene de promediar las cifras: $101.5 ; 102$ y 102.8. Los demás valores se obtienen con los mismos criterios de cálculo y se observa que no existen los dos primeros datos de la columna de valor promedio debido a que se requieren 3 datos como mínimo para obtenerlos. Una vez que se obtienen los datos del valor promedio de los últimos 3 días se realiza el gráfico correspondiente (Ablanedo, G., 2017).

Tabla 1

Datos aleatorios para ejemplificar la determinación de una media móvil simple.

$\begin{array}{cc}\begin{array}{c}\text { Preclos del activo } \\ \text { valoresaleatorlos) }\end{array} & \begin{array}{c}\text { Valor promedlo } \\ \text { (últimos 3dlas) }\end{array} \\ 101.0 & \\ 101.5 & \\ 1020 & 101.5 \\ 102.8 & 102.1 \\ 103.4 & 102.7 \\ 104.0 & 103.4 \\ 103.6 & 103.7 \\ 103.1 & 103.6 \\ 102.4 & 103.0 \\ 1020 & 102.5 \\ 101.7 & 1020 \\ 102.3 & 1020 \\ 102.5 & 102.2 \\ 103.0 & 102.6 \\ 103.9 & 103.1\end{array}$

Fuente: Elaboración propia, 2019.

La Tabla 1 permite identificar la manera de determinar una media móvil simple, para el caso de la media móvil exponencial que es la que se ocupó en éste trabajo, la fórmula se construye de la siguiente manera:

$M M E=(P K)+M M E$ (del día anterior) $(1-K)$

Donde: 
MME: Media móvil exponencial

$\mathrm{P}=$ es el precio de hoy, que puede ser de cierre, de apertura, etc, según elección del inversor. Cuanto más larga sea la tendencia que queremos medir, más datos necesita la media móvil.

$\mathrm{K}=$ es dos, dividido entre la suma de $\mathrm{N}+1 . \mathrm{K}=2 /(\mathrm{N}+1)$ Para un periodo como la media móvil exponencial de 5 días, sería 0.33 .

$\mathrm{N}$, es el número de periodos escogido para crear la media móvil.

Al final, se construyen 2 medias móviles que van a permitir identificar la tendencia del activo. El cruce de cada media móvil mostrará el cambio de tendencia y permitirá al inversor tomar posiciones largas (comprar porque el mercado es alcista) o cortas (vender porque el mercado es bajista). Expresado de otra manera, cuando la línea del promedio móvil permanece debajo de la línea de mercado es porque la tendencia es al alza, de igual forma, cuando la línea de la media móvil se encuentra por arriba del precio de mercado, es porque la tendencia es a la baja. La figura 2 muestra la manera en que el cruce de las medias móviles representadas por la línea continua roja y blanca, arroja señales para comprar o vender el activo.

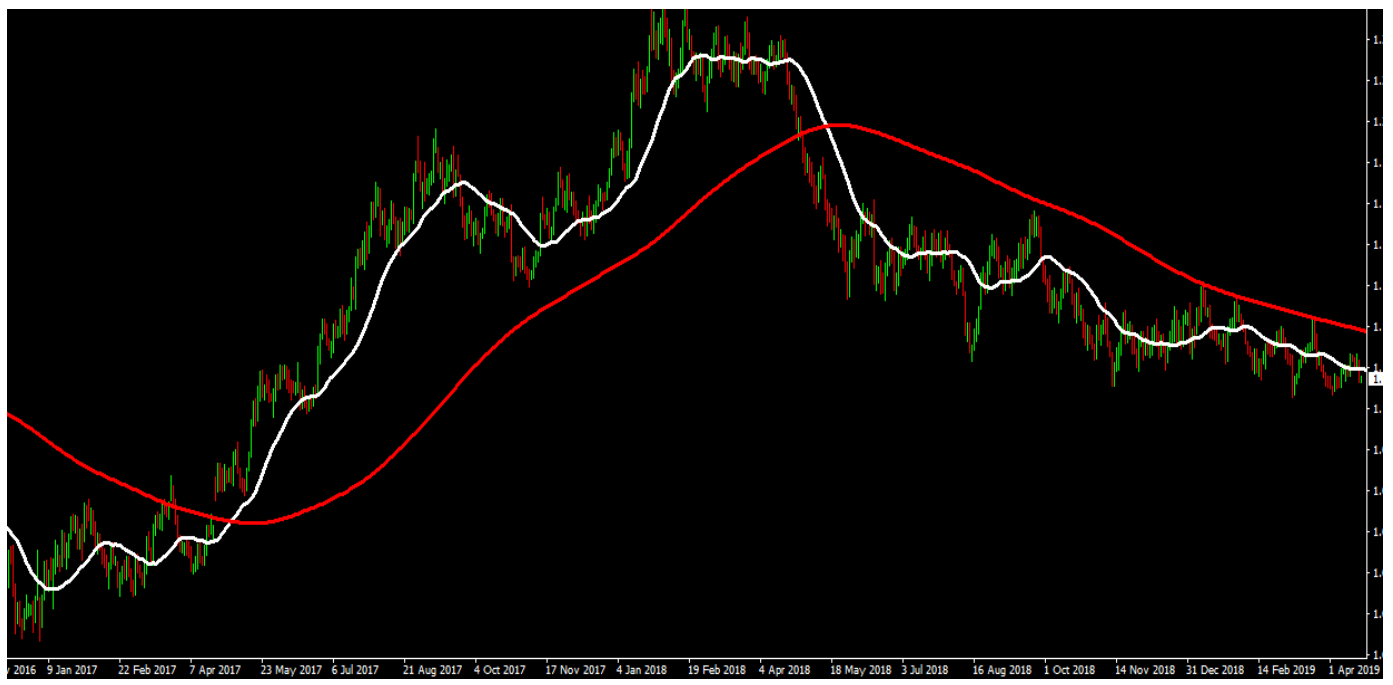

Figura 2. Cruce de medias móviles. Fuente: Elaboración propia incorporando datos de la plataforma Metatrader 4 (2018).

3. La media móvil de convergencia y divergencia es una herramienta diferente a la media móvil exponencial. Este indicador muestra la diferencia entre dos medias móviles exponenciales. Este indicador también se conoce como 
MACD y se mueven en torno a una línea en cero.

La figura 3 muestra en la parte inferior la representación del MACD y se aprecia que cuando la media móvil simple envía señal de cambio de tendencia, el MACD cruza la línea de 0 confirmando el cambio de tendencia.

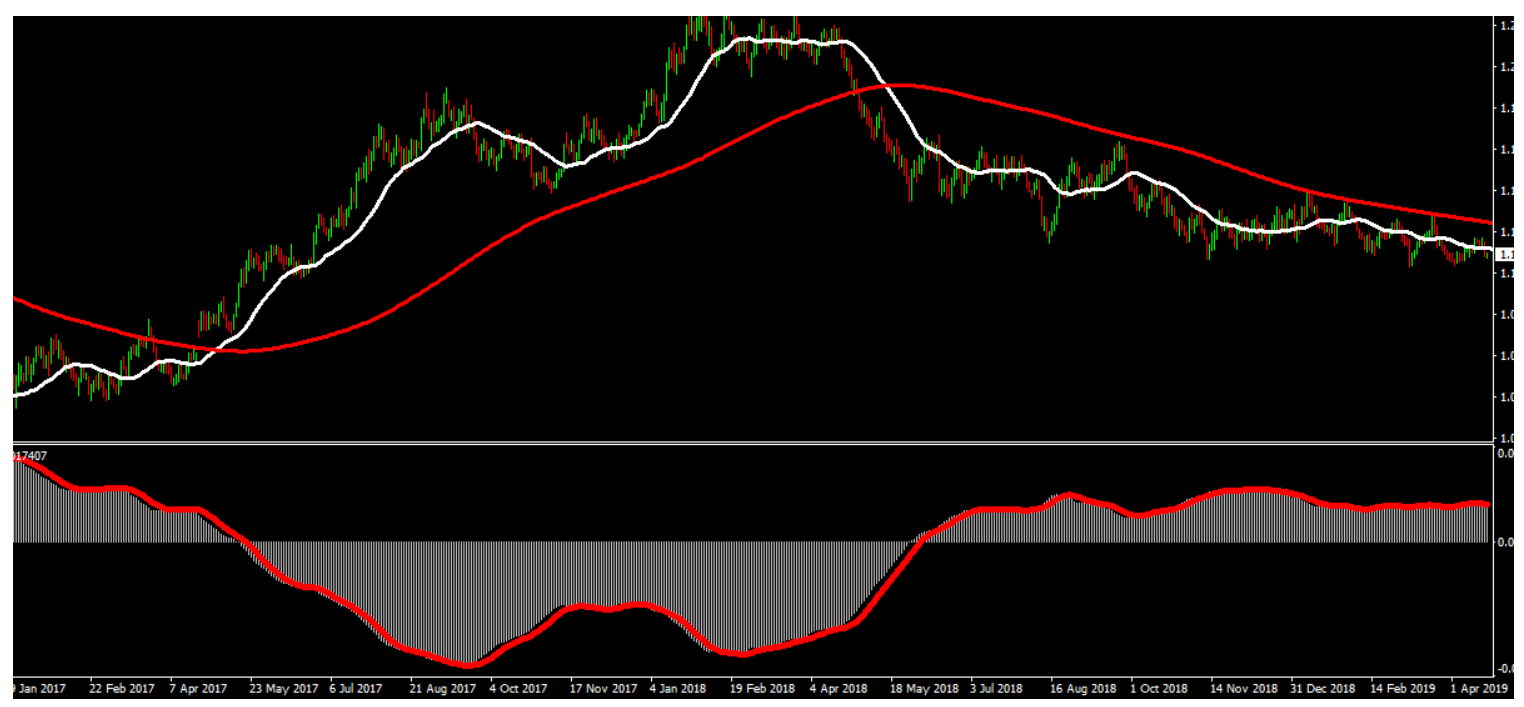

Figura 3. MACD en el cruce de medias móviles. Fuente: Elaboración propia incorporando datos de la plataforma Metatrader 4. (2018)

El objetivo de sobreponer el MACD de la figura 3 a la media móvil exponencial de la figura 2 tiene por objeto mostrar que las señales son muy similares, sin embargo, el MACD muestra divergencias que se refiere a que el indicador alcanza máximos más bajos y el precio alcanza máximos más altos. Esto indica que la fuerza del movimiento del precio se está agotando. Un ejemplo de esto se ve en la figura 4 y 5 donde la segunda curva de la parte inferior es más pequeña aun cuando el precio está alcanzando nuevos máximos. EI MACD puede ofrecer una señal adelantada de agotamiento de una tendencia.

Un inversionista, interesado en la bolsa, realizará análisis bursátil que consiste en la utilización de diversas técnicas de estudio para intentar predecir el valor de las acciones y la evolución de su precio (Díaz, 2013).

El enfoque técnico se basa en las siguientes premisas: Los cambios en los precios del mercado descuentan toda la información existente; los precios se mueven por tendencias y la historia se repite. Por regla, el inversor que usa el análisis técnico, no se ocupa de las razones por las que suben o bajan los precios. Pero usará los gráficos para identificar en qué dirección es más probable que se mueva el precio (Ablanedo, G., 2017). 
El chartista o inversor cuyas decisiones las basa en el análisis de precios que se plasma en los gráficos, sabe que hay razones por las que los mercados suben o bajan, pero no cree que sea necesario conocer esas razones para realizar su pronóstico (Serrano, 2013).

Técnicamente existen tendencias alcistas y bajistas, aunque es posible encontrar mercados laterales. Una tendencia en movimiento implica que es más probable que continúe a que retroceda, o bien, seguirá en la misma dirección hasta que comience a volver atrás (Sánchez, 2016).

En el comercio de activos bursátiles y en el análisis de software y gráficos, el período de tiempo se refiere a una periodicidad específica, la acción de precio se muestra en un gráfico donde cada vela representa un período determinado por el tiempo. Es decir, para una barra de 15 minutos, la vela se forma por la oferta y demanda durante dicho periodo antes de que empiece a formarse la siguiente. Así, en la gráfica, cada vela muestra quince minutos de acción de precio. Es común observar en un software gráficos de 1, 5, 15 y 30 minutos; 1 y 4 horas y finalmente el gráfico diario y semanal. El periodo a usar depende de las necesidades del inversor. Algunos inversionistas (scalpers) realizan operaciones de muy corto plazo apoyándose en gráficos de 1,5,15 o 30 minutos, mientras que otros realizan inversiones de mayor plazo apoyándose en gráficos de 4 horas y diarios (Ablanedo, G. 2017). Este trabajo se basa en gráficos con periodos diarios de tiempo.

El factor tiempo resulta ser un elemento crucial en el campo de decisión (Brito, 2016). La longitud más adecuada para una media móvil no existe, porque todas pueden tener su importancia y dependerán de la volatilidad del activo a analizar y de la periodicidad en la que se quiera invertir (Álvarez, 2013).

\section{V.- METODOLOGÍA, TÉCNICAS Y MATERIALES EMPLEADOS}

La descripción general de la metodología es el análisis estadístico de series de datos cuantitativos, sustentado en el software de Metatrader 4, cuyo producto será un informe referente a la recopilación, procesamiento y análisis de cifras numéricas que permitirán concluir sobre la incorporación de medias móviles exponenciales en estrategias de trading en el índice accionario en cuestión. No se puede trabajar en otra plataforma, es decir, existen otros proveedores de información, pero la serie de datos no reúne todos los requisitos de análisis técnico que si presenta Metratrader 4. Este software es uno de los más usados y de mayor importancia en los mercados financieros. Por eso se consideró como proveedor de información, generador de medias móviles, corredor de backtesting y generador de beneficios para el presente trabajo. Los gráficos no pueden ser tan visuales si no se presentan o generan mediante este proveedor. 
MetaTrader 4, también conocido como MT4, es una plataforma de comercio electrónico ampliamente utilizada por los comerciantes especulativos de divisas en línea. Fue desarrollado por MetaQuotes Software y lanzado en 2005. El software se autoriza a los corredores de divisas que proporcionan el software a sus clientes mismos que consta de un componente cliente y uno servidor. El componente del servidor es ejecutado por el corredor y el software del cliente se proporciona a los clientes del corredor, que lo utilizan para ver los precios de transmisión en vivo y gráficos, para realizar pedidos y para administrar sus cuentas.

Se importarán los datos del índice accionario de Singapur desde Metatrader 4 correspondientes a periodos de 10 años (1 de enero 2009 a 1 de abril 2019). La migración de los datos se realizó por medio de la creación de un script desarrollado en lenguaje MQL4 para evitar el error humano en la migración.

A los datos importados se les aplicarán distintos valores de medias móviles, cuando el precio cruza la media móvil se establece la estrategia "buy and hold" hasta que el precio vuelve a cruzar la media móvil. Se entiende como estrategia "buy and hold" comprar y mantener la posición hasta el final del periodo (en este caso, hasta el cruce de la media móvil). Por otro lado, cuando el precio cruza la media móvil de arriba hacia abajo, entonces se cierra la posición, pero ahora se abre una posición corta, para tratar de ganar en la caída del precio y se mantiene la posición hasta que la media vuelve a cruzar el precio en la dirección contraria (cerrando la posición corta y abriendo la larga).

Se correrá un backtest o proceso de revisión de la estrategia sobre cifras históricas, para identificar los parámetros que cumplen con la cobertura deseada o, en su defecto, que ofrezcan buenos niveles de confianza. Para evaluar la solución se programa un sistema de trading utilizando lenguaje de programación MQL4 y se compara su rentabilidad con los distintos parámetros utilizando marcos temporales diarios.

Se generan resultados a las siguientes estrategias:

a) Medias móviles exponenciales cuyo cruce de abajo hacia arriba representa señal de compra y viceversa. No existe Stop Loss o take profit debido a que el cruce cierra una posición para abrir otra. Los parámetros que se consideraron fue una media móvil de 210 sesiones como periodo mayor y una media móvil de 35 sesiones de periodo menor. Sus cruces son los que generan cambios de tendencia y los que ocasionan ganancias o pérdidas.

b) MACD cuyo cruce en la línea cero representa señal de compra y viceversa. Tampoco se establece Stop Loss o take profit debido a que el cruce cierra una posición para abrir otra. Igualmente se consideraron los parámetros de 210 
sesiones para el periodo mayor y de 35 sesiones para el periodo menor.

c) MACD cuyo cruce en la línea cero representa señal de compra y viceversa. Pero también, cuando la media móvil se encuentra por dentro o por fuera de cada una de las líneas verticales del histograma. Tampoco se establece Stop Loss o take profit debido a que el cruce cierra una posición para abrir otra. Nuevamente los parámetros son de 210 sesiones y de 35 sesiones.

El objetivo de mantener los mismos parámetros de 210 y 35 sesiones era tratar de observar los resultados sin alterar el comportamiento de las medias y así poder tener datos comparativos sobre ambas. Es importante resaltar que en libros y revistas se realizan pruebas con parámetros diferentes que generan resultados diferentes, pero evidentemente no comparables.

Para comprender más la diferencia entre la estrategia del inciso b y la del inciso c se muestra la siguiente figura 4 que muestra que por la estrategia B solo existen 2 momentos de cambio de tendencia, mientras que en la $C$ existen esos 2 y otros más que se representan por el cruce de la media móvil dentro del histograma representado por líneas verticales. Es decir, aun cuando los parámetros son los mismos, el MACD es un indicador poco más robusto y complejo que ofrece más señales de entrada y salida mismas que fueron consideradas en éste trabajo. Los resultados de este trabajo demuestran que no suele ser un buen indicador cuando un activo se encuentra en lateralidad, para muchos, lo importante de este indicador que se basa en medias móviles exponenciales es precisamente identificar las convergencias y divergencias en los precios de un activo tal como lo señala su nombre. 


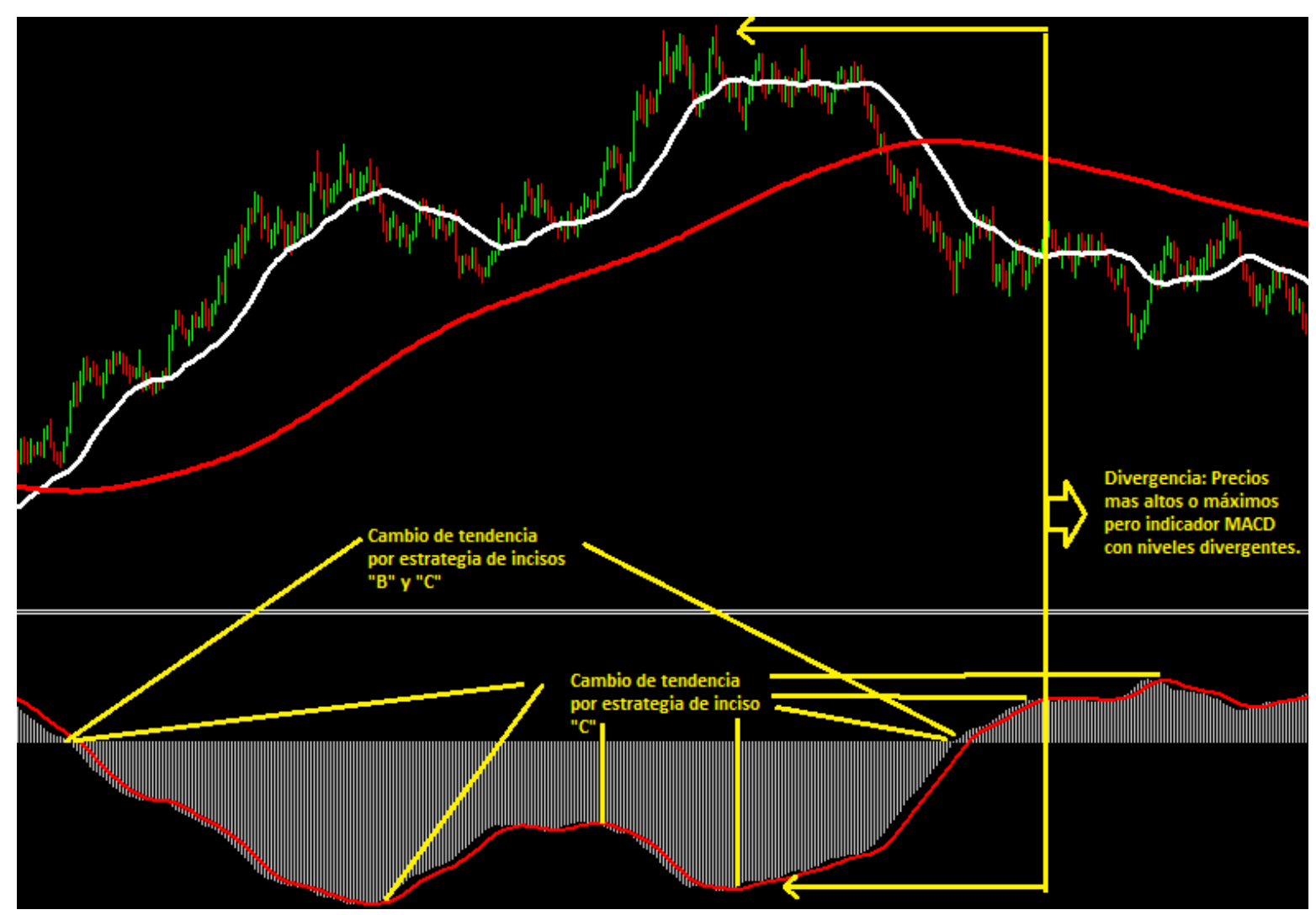

Figura 4. Gráfico de las tendencias expuestas en incisos b y c. Fuente: Elaboración propia incorporando datos de la plataforma Metatrader 4. (2018)

\section{VI.- $\quad$ RESULTADOS}

Códigos de interpretación:

- El campo 'Barras en el historial' muestra la profundidad de datos en que se ha basado el modelado.

- El campo 'Ticks modelados' recientemente ha tomado importancia entre los traders y cada tick se refiere a cada cotización realizada. A más actividad, más ticks y por tanto mayor movimiento. Aquí el volumen juega un papel muy importante. Con esta estrategia los operadores afirman que lo importante no es el tiempo, sino las operaciones o periodos de inactividad que se realizan.

- La calidad del modelado se refiere a obtener una buena calidad de modelado (99\%). Para confiar más en los resultados del backtest y optimizaciones, lo mejor es usar alguno de los productos que permiten hacer backtest tick a tick como: Ticksory o TDS (tick data suite). El programa que acá se usó, no se basa en tick sino en temporalidades, periodos o timeframes de 1 día.

- El beneficio bruto es la suma de las ganancias de todas las transacciones 
rentables durante el periodo de prueba. Todos los resultados descritos en éste apartado, se obtienen tras simular la inversión de 10,000 dólares para posiciones largas y cortas tras el cruce del precio con la media móvil.

- La pérdida bruta es la suma de las pérdidas de todas las transacciones perdedoras durante el periodo de prueba.

- El beneficio neto total es la diferencia entre el beneficio bruto y la pérdida bruta.

- El factor de beneficio muestra la relación entre el beneficio bruto y la pérdida bruta, un buen resultado arrojaría un valor superior a 1.

- El Beneficio esperado se calcula de la siguiente manera:

Beneficio esperado $=$ (Beneficio bruto / Total de transacciones) - (Pérdida bruta / Total de transacciones).

Una buena estrategia debería arrojar un resultado positivo en este indicador.

- El Drawdawn máximo y absoluto refleja la pérdida más alta registrada en el periodo, su análisis puede dar la idea del monto mínimo que se requiere al momento de abrir una cuenta.

- El total de operaciones corresponde al total de transacciones que se realizaron en la periodicidad señalada.

- Las posiciones cortas (ganadoras \%) y las posiciones largas (ganadoras \%) se refiere a la cantidad total de posiciones cortas o largas y el porcentaje de operaciones rentables existente entre las mismas (posiciones cortas o largas rentables / cantidad total de posiciones cortas o largas * $100 \%)$.

- Transacciones rentables (\% del total) resulta de dividir la cantidad total de transacciones rentables entre el total de transacciones. Debido a que cada operación de apertura y cierre implica una comisión, es importante tener pocas operaciones, pero con mayor porcentaje de acierto.

- Transacción rentable mayor se refiere a la operación rentable más grande de todas las transacciones, mientras que la transacción no rentable mayor se refiere a la pérdida más grande de todas las transacciones. Se sugiere que el inversionista incorpore estrategias de detención de pérdida (Stop Loss, o bien, SL por sus siglas en inglés), lo que implicaría con seguridad, mejorar los beneficios en la inversión.

- La media de transacciones rentables se refiere al tamaño medio de las operaciones rentables; por otro lado, la media de transacciones no rentables, calcula el tamaño medio de las operaciones perdedoras.

- El máximo de ganancias consecutivas (beneficios en dinero) representa la cantidad máxima de ganancias consecutivas en la serie de operaciones rentables y la suma del beneficio de la serie; mientras que el máximo de pérdidas consecutivas (pérdidas en dinero) señala la cantidad máxima de pérdidas consecutivas en la serie de operaciones perdedoras y la suma de las pérdidas de la serie. 
- El máximo de beneficios consecutivos (número de ganancias) se refiere al beneficio máximo de la serie consecutiva de operaciones ganadoras y la cantidad de operaciones de la serie; mientras que el máximo de pérdidas consecutivas (número de pérdidas) se refiere a la pérdida máxima de una serie consecutiva de operaciones perdedoras y la cantidad de operaciones de la serie.

- El promedio de ganancias consecutivas se refiere a la cantidad media de operaciones de la serie de beneficios consecutivos. Por otro lado, el promedio de pérdidas consecutivas se refiere a la cantidad media de operaciones de la serie de pérdidas consecutivas.

Se corre la prueba de la estrategia del inciso "a" del apartado anterior aplicando la media móvil exponencial a gráficos diarios considerando media móvil larga de 210 sesiones y la corta de 35 . En la figura 5, se exponen el resultado e interpretación correspondientes. La razón por la que se tomaron estos valores tiene que ver con que previamente, antes de realizar éste trabajo, se hicieron pruebas exclusivamente con medias móviles simples para determinar cuáles valores son los que arrojan los mejores beneficios. En dicho test, se encontró que, para el índice accionario de referencia, las medias móviles que se ajustaban a los cambios en los precios y que ofrecían resultados aceptables eran las de 210 y 35 sesiones. Lo anterior no quiere decir que estos parámetros son los que deban aplicarse a todos los activos financieros, cada activo financiero se adaptará a una media móvil diferente en función al comportamiento del precio. Cada inversor debería realizar un análisis previo antes de identificar con qué medias móviles trabajará.

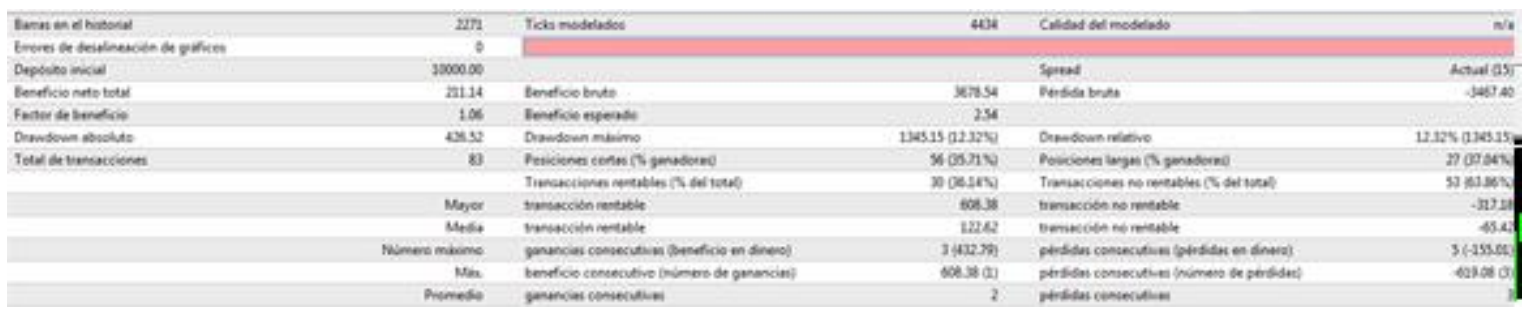

Figura 5. Resultados de aplicar media móvil exponencial de 210 y 35 sesiones. Fuente: Elaboración propia incorporando datos de la plataforma Metatrader 4. (2018)

El siguiente gráfico muestra el comportamiento de los rendimientos correspondientes a la estrategia de media móvil exponencial considerando la sesión larga de 210 sesiones y la corta de 35 . 


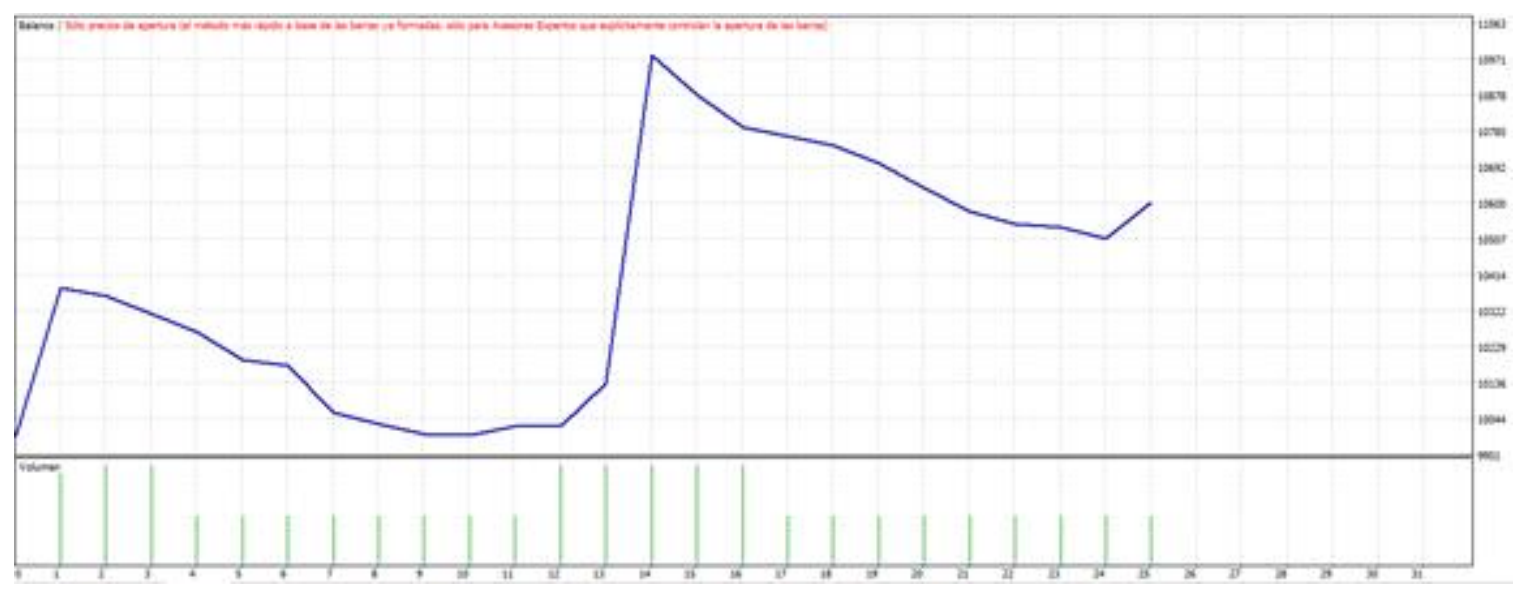

Figura 6. Beneficios para medias móviles exponenciales de 210 y 35 sesiones. Fuente: Elaboración propia incorporando datos de la plataforma Metatrader 4 (2018).

Como se aprecia, el beneficio corresponde a una ganancia del $6 \%$ para el periodo analizado. Se observa que dicho beneficio no es atractivo aun cuando se encuentra representado en dólares. Por esa razón, se trata de buscar alternativas que traten de maximizar dichos beneficios.

Por otro lado, se corre la prueba del inciso "b" al que se refiere el apartado de metodología donde se analiza la media móvil de convergencia y divergencia, cuyo cruce en la línea cero representa señal de compra y viceversa. Aplicando parámetros de 210 sesiones para la media larga y 35 sesiones para la corta y los resultados, como eran de esperarse, son casi los mismos debido a que el MACD se integra de Medias móviles exponenciales. Por lo anterior, se omite presentar los resultados de forma gráfica y numérica, aunque se sí se menciona que el beneficio fue de $6.01 \%$. La diferencia es mínima y no tiene caso realizar un análisis mayor en este sentido.

Finalmente se corre la prueba faltante correspondiente al inciso " $c$ " del apartado de metodología donde se analizan los resultados correspondientes al cruce en la línea cero del MACD y también, cuando la media móvil se encuentra por dentro o por fuera de cada una de las líneas verticales del histograma. Desde luego que los resultados deben variar en función a que habrá más señales de entrada y salida que las que se presentan en para exclusivamente para las medias móviles exponenciales. Es importante señalar que tantas señales repercuten en costos financieros para el inversor. 


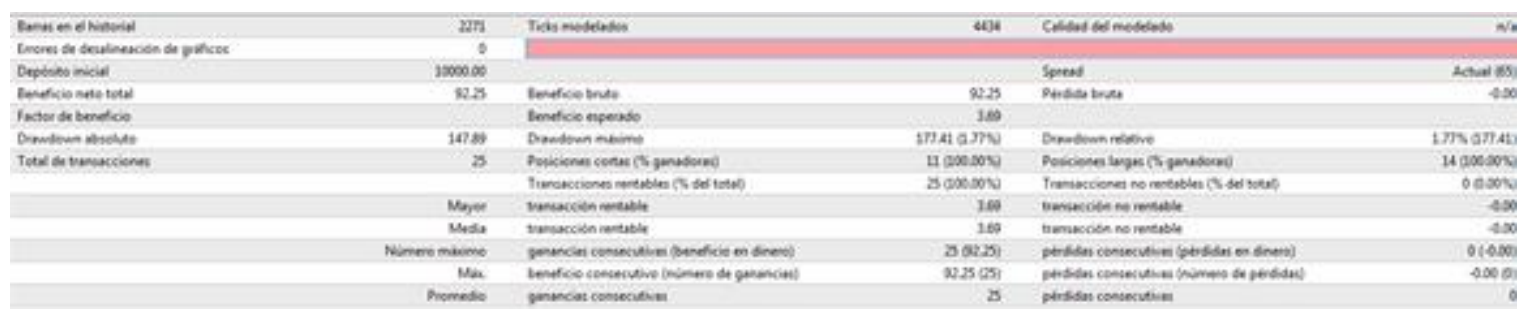

Figura 7. MACD en línea cero y en cruce de media móvil dentro del histograma. Fuente: Elaboración propia incorporando datos de la plataforma Metatrader 4 (2018).

El siguiente gráfico muestra el comportamiento de los rendimientos correspondientes a ésta estrategia.

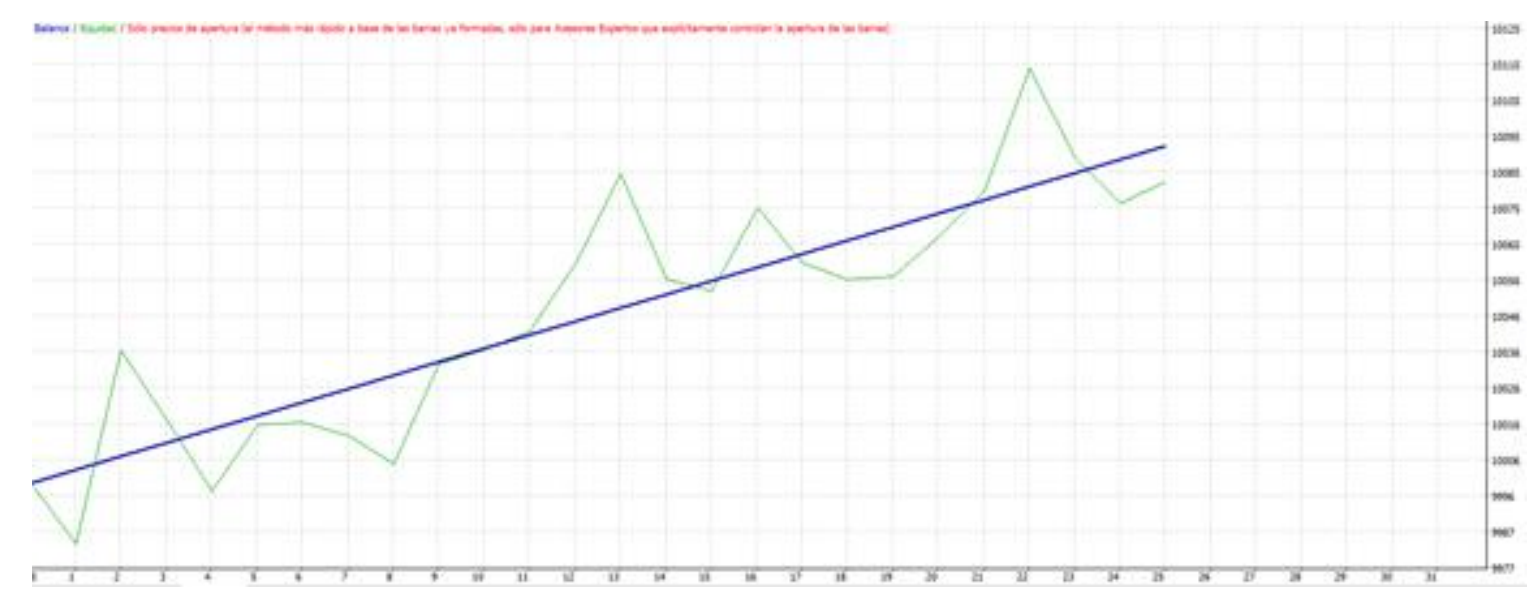

Figura 8. MACD en línea cero y en cruce de media móvil dentro del histograma. Fuente: Elaboración propia incorporando datos de la plataforma Metatrader 4. (2018).

El beneficio corresponde a una ganancia del 1\% para el periodo analizado. Que implica un beneficio muy bajo para todo el periodo de análisis y quizás frustrante para un inversor que aplicó la técnica durante todo este tiempo y solo se llevó dicho beneficio.

Igualmente, los resultados muestran que la estrategia "Buy and hold" que se refiere a comprar y mantener, ofrece beneficios atractivos por el tiempo analizado (1 de enero 2009 a 1 de abril 2019). Los valores que corresponden a ésta técnica se reflejan en la figura 1 y el rendimiento que corresponde al periodo de análisis corresponde a un $11 \%$ de beneficio. El dato previo tampoco refleja un rendimiento muy atractivo para el periodo de análisis (aun cuando se obtiene en dólares americanos), y es precisamente la razón por la que algunos inversores o especuladores intentan identificar nuevas técnicas que incrementen los beneficios tras el alto riesgo que esto implica. 
Para algunos, la estrategia de comprar y esperar es una de las mejores opciones al momento de invertir, Es importante señalar que dicha estrategia paga también dividendos pues el inversor adquiere las acciones y los derechos que de ella emanan, Es importante señalar que en este trabajo no se están reflejando los beneficios que corresponden a los rendimientos de las emisoras que integran el índice de Singapur, por lo que el resultado del $11 \%$ mencionado en el párrafo previo, podría ser ligeramente superior al momento de considerar dichos valores. Para los que se dedican a la inversión, ésta estrategia no suele ser la preferida precisamente porque implica mucha pasividad y los inversores que están en bolsa buscan ser más activos con la intención de mejorar sus expectativas de beneficio. El resultado de invertir en la estrategia "Buy and hold" por el mismo periodo de análisis que para las medias móviles se muestra en la siguiente figura:

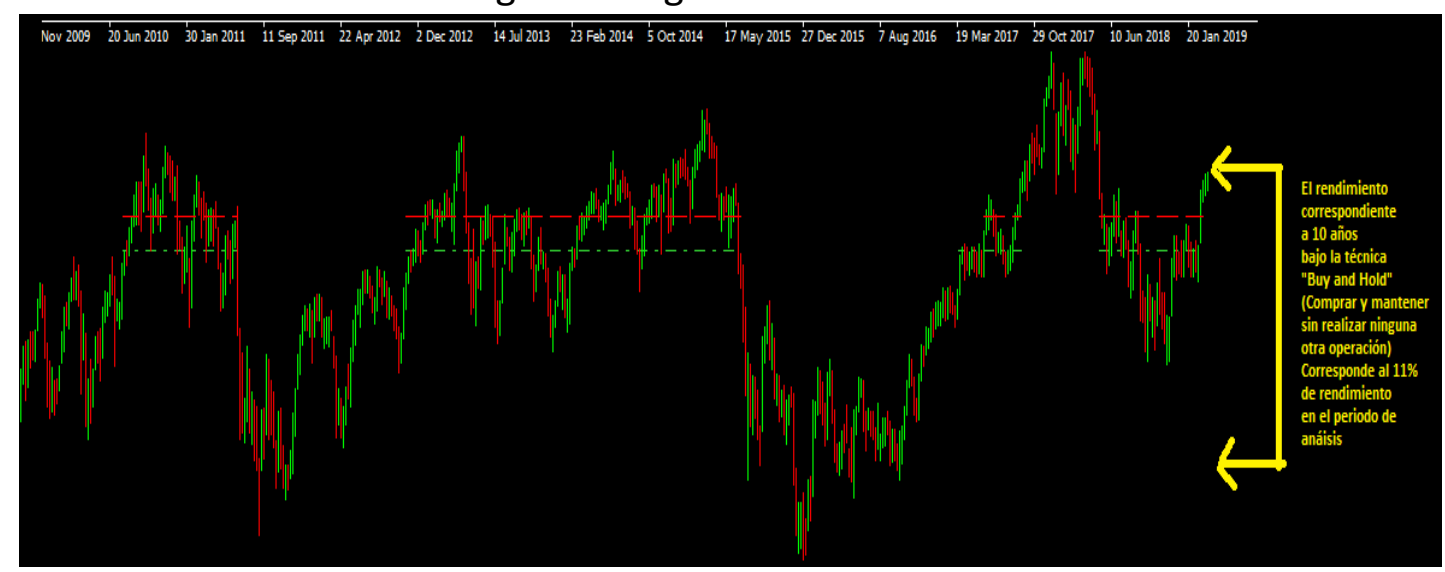

Figura 9. Precios históricos de 10 años y beneficio de estrategia "Buy and Hold". (2018)

\section{VII.- DISCUSIÓN DE LOS RESULTADOS}

Las medias móviles representan un indicador que permite verificar la existencia de una tendencia, pero no siempre son indicadores que permiten maximizar beneficios. En éste trabajo se analizan los resultados de comprar y vender cada que se presenta el cruce de la media móvil y los resultados arriba relacionados, muestran que no es una buena idea realizarlo. La razón de lo anterior tiene que ver que existen varios cruces de medias que indican cambios de tendencia, pero éstas no suelen ser tan largas lo que implica que se tenga que entrar y salir varias veces de la posición. En ausencia de comisiones como es el caso de éste análisis, los resultados como ya se mostró, no son los óptimos. Si además, se considera que por cada entrada y salida, es decir, cada cambio de tendencia implica cerrar una posición para abrir una contraria, entonces las cargas financieras por cada transacción impactan en los beneficios. Estos costos financieros no aplican cuando se trata de comprar un activo y mantenerlo por largo plazo (Buy and hold).

Por lo que se observa en los resultados, éstos indicadores permiten identificar la 
consistencia de la tendencia y son una herramienta de verificación de cambio de tendencia. Sin embargo, otro problema de la media móvil radica en que puede obtenerse del número de días que el inversor desee, pero cada activo es diferente en cuando a volatilidad y tendencia lo que implica que existirá una media móvil "ideal" para cada caso particular.

Otro de los elementos de juicio a considerar en función a los resultados tiene que ver con que el indicador MACD, por su complejidad, arroja muchas más señales de entrada y salida que la media móvil exponencial, desde luego que esto hace que los resultados de aplicar una u otra varíen tal como acá se demostró.

Posiblemente, una alternativa viable del poder predictivo, sería correr varios backs testing como los que en este trabajo se realizaron, pero no solo correrlos en escenarios de un día, sino correrlos en timeframes de 5, 15, 30, 60, 120, 240 y 720 minutos para observar si en alguna temporalidad los resultados se optimizan. Esto puede llevar mucho más tiempo e inclusive en plataformas sofisticadas como las de Metatrader es casi imposible correr esta prueba para 10 años de análisis por la cantidad de datos que se requieren y que no están disponibles en la plataforma.

Existen versiones encontradas referentes a que si el análisis técnico es una buena opción o no. Inclusive, el profesor Eugene Fama (mejor conocido por su trabajo sobre la fijación de precios de activos y la teoría de los mercados eficientes) o Burton Malkiel (famoso por su clásico libro sobre finanzas "Un paseo aleatorio por Wall Street"). Sugieren que tratar de adivinar o identificar la tendencia de los precios es imposible y sostienen que los mercados reflejan el valor justo del activo en cuestión. Esto último es lo que se está demostrando en este trabajo de investigación.

Continuando con la discusión de resultados de este trabajo, se vuelve interesante la pregunta académica que surge cuando en aula se explica la teoría del análisis técnico, cuyo primer cuestionamiento tiene que ver con identificar el número de personas que se han logrado beneficiar considerando el análisis técnico en su estrategia de inversión. Y la respuesta ampliamente compartida por los interesados e investigadores del tema, es que no existe ninguna persona en el mundo que se haya hecho rico usando ésta estrategia. Inclusive, estudios serios mencionan que la especulación a corto plazo con éste tipo de herramientas tiene que ver más con probabilidades y suerte que con una buena técnica de inversión. Esto es precisamente lo que en este trabajo se demostró de manera muy breve y general. No obstante, la duda surge cuando eventualmente, la aplicación de esta estrategia sí da resultados como fue el caso de los últimos 2 años en el par dólar americano-peso mexicano. Siendo así, y en apego a un apartado de discusión que se pide como requisito para este trabajo, podemos afirmar que pase lo que pase siempre habrá algo que permita justificar una u otra técnica. Pero siempre va a ser importante que, al momento de adoptar cualquier estrategia, se justifique o sustente su adopción en función a un análisis cuantitativo como el que acá se 
desarrolló.

\section{CONCLUSIONES}

Este trabajo responde a la pregunta de investigación referente a la mejor estrategia para invertir considerando 3 alternativas: Media móvil exponencial, media móvil de convergencia y divergencia, estrategia "buy and hold" o comprar y mantener. Se observa que de acuerdo a los resultados que se generan, no siempre el análisis técnico es la mejor estrategia de inversión. De hecho, es solo una herramienta de apoyo, pero no se recomienda su uso sistemático.

También se responde a los dilemas planteados. Se confirma que, en el caso de esta investigación, la estrategia más apropiada era la de comprar y mantener, posteriormente la media móvil exponencial y finalmente MACD. Pero también se afirma los resultados pueden variar dependiendo de la volatilidad del activo a analizar y de los marcos de temporalidad en los que se quiera invertir. Se sugiere realizar más pruebas considerando éstos 2 elementos.

Otro de los dilemas se refiere a los inconvenientes de aplicar la estrategia más rentable, en cuyo caso se expuso que la problemática radica en las comisiones que se generan por entrar y salir en el mercado. Es decir, cada que la media móvil exponencial o la media móvil de convergencia y divergencia ofrecen señales de tendencia alcista o cambio de tendencia a una bajista, el inversor debería estar realizando éstas operaciones con las consecuentes cargas financieras que esto ocasiona. Por lo anterior, los inconvenientes radican en el número de operaciones a realizar con éstas técnicas, por lo que posiblemente se requiera de otros indicadores que permitan confirmar las tendencias y eviten entradas y salidas en falso lo que implicaría disminuir costos de transacción. Igualmente se afirma que en el caso de "buy and hold", este inconveniente no existe.

Según los resultados, se afirma que las medias móviles no superan constantemente la estrategia "buy and hold", lo que confirma que solo se trata de una herramienta de análisis mas no se sugiere usar como técnica de inversión.

Finalmente, se concluye que el inversor debe considerar el análisis técnico como una herramienta, mas no se sugiere realizar sistemáticamente operaciones con algunos elementos de dicho análisis. El trabajo demuestra que robotizar operaciones considerando las medias móviles podría implicar que los beneficios se modifiquen en contra del inversor.

\section{REFERENCIAS}

Ablanedo, G. (2017). Inclusión y optimización de medias móviles para predicción de tendencias en la divisa dólar americano-peso mexicano. CUMEX, 541-555

Álvarez, C. R. (2013). Finanzas amigables. México: Trillas, 25-28 
Blázquez, B. H. (2014). Compendio bursátil. España: Ediciones Diaz de Santos, 99-105

Brito, G. A. (2016). Finanzas Bursátiles. México: IMCP.

Díaz, H. E. (2013). Finanzas y bolsa, diccionario bursátil. Evaroz.

Maidona, A. D. (2016). Bolsa Argentina. Sistemas de trading exitosos. Argentina: Ediciones B.

Rodríguez, M., \& García Fronti, J. (2013). Finanzas Personales, acciones y bonos. Alfaomega.

Sánchez, G. J. (2016). Manual básico de trading. España: Kindle.

Serrano, F. G. (2013). Escuela de Bolsa. España: Almuzara. 